\title{
Systemic Inflammatory Response Syndrome (SIRS) in the emergency department: An original investigation - A look back in time
}

\author{
Andrew H Bissonette ${ }^{1}$, Alan P Tuttle ${ }^{2}$, Mary Grzybowski ${ }^{1}$, Richard M Nowak ${ }^{1}$, Douglas S Ander ${ }^{3 *}$, Daniel C Morris ${ }^{1}$, Deanna Minisee-Ryce ${ }^{1}$ \\ and Emanuel P Rivers ${ }^{1}$ \\ ${ }^{1}$ Department of Emergency Medicine, Henry Ford Hospital, Detroit, MI, USA \\ ${ }^{2}$ Department of Emergency Medicine and Division of Pulmonary, Allergy, and Critical Care Medicine and Department of Internal Medicine, University of New \\ Mexico-Health Sciences Center, Albuquerque, USA \\ ${ }^{3}$ Department of Emergency Medicine, Emory University School of Medicine, Atlanta, GA, USA
}

\begin{abstract}
Background: Characterize the clinical and prognostic importance of the systemic inflammatory response syndrome (SIRS) in an emergency department (ED).

Methods: Prospective observational study with follow-up until hospital discharge. Triage in an ED of an urban tertiary health care institution. All consenting adult ( $>18$ years of age) patients presenting to the ED over a 2 week period. SIRS criteria were assessed in all participants. Admission rate, length of stay, and hospital charges.

Results: During the study period, there were 1148 distinct ED patient visits, of which 991 patients participated. Of these, 247 (24.9\%) met criteria for SIRS defined as the presence of $>2$ SIRS criteria. Patients with SIRS had higher admission rates ( $41.3 \%$ vs. $13.6 \%$, p $<0.001)$, longer lengths of ED stay $(8.0 \pm 5.9$ vs. $6.0 \pm 4.6 \mathrm{~h}$, $\mathrm{p}<0.001$ ), and higher ED charges ( $\$ 463 \pm 30$ vs. $\$ 283 \pm 11, \mathrm{p}<0.001)$. If admitted, patients with SIRS in the ED had longer inpatient stays (8.7 \pm 12.0 vs. $4.5 \pm$ 4.6 days, $p<0.004)$, increased service costs $(\$ 30,305 \pm \$ 3000$ vs. $\$ 11,362 \pm \$ 1130, p<0.002)$, and trended towards increased procedural charges $(\$ 5326 \pm \$ 527$ vs. $\$ 3204 \pm \$ 320, \mathrm{p}<0.067$ ). Additional SIRS criteria present at entry led to higher admission rates and longer ED and inpatient stays. The sensitivity, specificity, positive predictive value, and negative predictive value of admission to the hospital for those meeting criteria for SIRS were $50.3 \%, 81.6 \%, 41.3 \%$, and $86.4 \%$, respectively. Of patients meeting criteria for SIRS, 59\% were discharged home from the ED.
\end{abstract}

Conclusion: The presentation of patients with SIRS in the ED is common. Patients presenting to the ED meeting criteria for SIRS utilize greater health care resources than their non-SIRS counterparts, but SIRS is a poor predictor of the need for hospitalization.

Abbreviations: ACCP: American College of Chest Physicians; CI: Confidence Interval; ED: Emergency Department; ICU: Intensive Care Unit; LOS: Length of Stay; NINT: Noninfectious and Nontraumatic; SCCM: Society of Critical Care Medicine; SD: Standard Deviation; SIRS: Systemic Inflammatory Response Syndrome

\section{Introduction}

SIRS is the physiologic state of hypermetabolism resulting from a variety of host insults. It is clinically manifested by, but not limited to, 1 or more of the following criteria: 1) tachycardia, heart rate $\geq 90$ beats/min; 2) tachypnea, respiratory rate $\geq 20$ breaths/min or Pco $2 \leq$ $32 \mathrm{~mm} \mathrm{Hg}$; 3 ) thermal dysregulation, temperature $\geq 38^{\circ} \mathrm{C}$ or $\leq 36^{\circ} \mathrm{C} ; 4$ ) leukocytosis/leukopenia, white blood cell count $\leq 4$ or $\geq 12 \times 109 / \mathrm{L}$ or $\geq 10 \%$ immature neutrophils (band forms) [1]. SIRS has been shown to be the first step in the hierarchical continuum of the inflammatory response that leads to multi-organ dysfunction syndrome and death [2,3].

The concept of SIRS was first described in the landmark ACCP/ SCCM Consensus Conference of 1992. The conference was convened in an effort to clarify the ambiguous terminology applied to the immune response at the time. Since that time, SIRS has been described in association with a wide range of clinical states, the best described of which is infection [4-6]. SIRS may also be a manifestation of trauma, $[7,8]$ pancreatitis, $[1,8]$ burns, $[1,8]$ or cardiac surgery [9].

Although the definition of sepsis reflected use in the intensive setting, the expanded use in the ED setting was unknown. Although over $50 \%$ of patients presenting to the ICU originated in the ED, the venue was not previously examined. Thus, the diagnosis of sepsis provided unique and fundamental challenges to the ED. The use of concepts such as SIRS were functional for critical care medicine but were unknown for the ED setting. This question provided the original scientific investigation for the use of SIRS in the ED setting. As a result, this publication was the stimulus for changing the early diagnosis and treatment of sepsis.

${ }^{\star}$ Correspondence to: Douglas S Ander, Department of Emergency Medicine, 49 Jesse Hill Jr Doctor Atlanta, GA 30303, USA, E-mail: dander@emory.edu

Received: November 12, 2018; Accepted: November 20, 2018; Published: November 23, 2018 
This paper represents an examination of sepsis that was previously published in abstract but represents one of the fundmental changes in the diagnosis and treatment of sepsis in the ED setting.

\section{Materials and Methods}

\section{Study Subjects}

From November 28, 1995 to December 4, 1995 and from December 6 to December 12, 1995, 1148 adult patients (age $>18$ years) were recruited consecutively between the hours of $10 \mathrm{AM}$ and $10 \mathrm{PM}$ during triage in the ED at Henry Ford Hospital in Detroit, Michigan, an urban level-I trauma center with an annual ED volume of 90,000 patient visits. Patients receiving medical stabilization from another health care facility prior to presenting to the ED, patients in cardiac arrest, or patients presenting to the ED for repeat visits within the study period were not included. The Institutional Review Board of Henry Ford Hospital approved all aspects of this study.

Eligible patients were evaluated at triage by registered nurses that were familiar with the study protocol. A senior staff physician at triage obtained verbal consent. Consent was assumed by institutional review board waiver for patients unable to provide verbal consent. Participants answered 15 questions regarding their medical status and donated 20 $\mathrm{ml}$ of blood at triage.

\section{Data Collection}

Date and time of ED presentation, general acuity status, vital signs, and demographics were assessed and recorded. Follow-up data collected included ED discharge information (date, time, and diagnosis) and ED disposition status (discharge home or hospital admission). If admitted to the hospital, admission unit (intensive care, telemetry/step-down unit, general medical/surgical ward, or operating room) and hospital discharge information (date, time, and diagnoses) were collected via medical chart review and computerized hospital information database. SIRS was defined according to the definition published by the ACCP/SCCM Consensus Conference [1]. Charge data on all participants were obtained from Henry Ford Hospital's Fee Audit Services and Corporate Billing Departments. Specifically, service (non-physician) and procedure (physician) charges were obtained. A maximum of the three primary ED discharge diagnoses were obtained, and each discharge diagnosis was subsequently subdivided into 3 disease categories: infectious, traumatic, or neither (i.e., noninfectious and nontraumatic [NINT]) by 1 of the investigators. The infected group consisted of patients with diagnoses related to infectious or presumed infectious etiology. Limited resources prevented us from validating all infectious diagnoses. Patients were classified as having a traumatic diagnosis if they were subject to any trauma prior to entry. All other patients were categorized as NINT.

Blood samples were obtained prior to the institution of therapy. Participating patients were not charged for any clinically unnecessary laboratory tests. Once obtained, $5 \mathrm{ml}$ of blood was sent to the pathology laboratory for complete blood counts and white blood cell differential analysis. All complete blood counts and white blood cell differentials were determined using Coulter Stacker S (Coulter International Inc., Hialeah, FL). Aberrant white blood cell differentials were flagged by the device and were subjected to manual slide determination. Remaining blood was immediately centrifuged at 3000 rotations/min for $10 \mathrm{~min}$ in a refrigerated centrifuge, and sera were stored in a biorepository at $-70^{\circ} \mathrm{C}$ for future serological studies.

\section{Data Analysis}

Summary statistics were used to describe the population. Continuous variables were assessed for normality using the ShapiroWilk statistic. Approximate normality was held for most variables. Logarithmic transformations were made as indicated by the data for variables not having normal distributions. All measurements are expressed as mean \pm standard deviation.

For comparisons among groups, two-sample, two-sided Student t-tests were used for normally distributed data, and the Welch's p-value was reported in the event of unequal standard deviations in the 2 groups. For comparisons among groups for non-normally distributed variables, the Wilcoxon signed-rank test was used. Comparisons among 2 or more groups were made using one-factor analysis of variance with Bonferroni pairwise comparisons.

Categorical data were analyzed by the likelihood chi-square technique with Yate's correction as indicated by the data. Fisher's exact test was used if the expected number of observations in any cell was less than 5. Odds ratios and 95\% confidence intervals (CI) were calculated. Least squares regression and the chi-square test for linear trends were used to assess trends in the data. To screen for the performance of hospital admission prediction, sensitivities, specificities, and positive predictive values were calculated for each of the number of SIRS criteria met (0-4). For all statistical analyses, a 0.05 level of significance was used, and all p-values were two-sided. All analyses were done using SAS 11.0 (SAS Institute Inc., Cary, NC).

\section{Results}

A total of $1148 \mathrm{ED}$ patients were eligible to participate in the study. Of these, 47 (4.1\%) were excluded from analysis, leaving a total of 1101 eligible patients for participation. Exclusion was due to the following: 30 patients $(63.8 \%)$ had at least 1 missing SIRS criterion, $12(25.5 \%)$ went to another health care institution, $4(8.5 \%)$ were in cardiac arrest, and $1(2.1 \%)$ left the ED against medical advice.

Of the 1101 eligible participants, 991 (90.0\%) consented to participate in the study. Reasons for refusal to participate included fear of needles (43.6\%), medical contraindications (9.1\%), disbelief in medical research $(8.2 \%)$, psychological reasons $(1.8 \%)$, and religious reasons $(0.9 \%)$. The remaining $36.4 \%$ did not provide rationale for declining participation. Participants differed from nonparticipants in terms of age and hospital admissions (Table 1). Participants were, on average, 7 years older than nonparticipants $(p<0.001)$ and were more frequently admitted to the hospital $(\mathrm{p}<0.001)$. Inpatient hospital costs were not significantly different between groups.

The participants' mean age was $47.0 \pm 20.1$ years with a range from 18 to 97 years. Of these subjects, $463(46.7 \%)$ were male, 135 (13.6\%) were white; an total of $203(20.5 \%)$ were admitted to the hospital, and 9 (4.4\%) died. The cost associated with the participants' ED visit was $36.7 \%$ higher than that of the nonparticipants $(\mathrm{p}<0.001)$; however, there were no significant differences between participants and nonparticipants regarding procedure or inpatient hospital charges.

The overall prevalence of SIRS (defined as the presence of $\geq 2$ SIRS criteria) was $24.9 \%(\mathrm{n}=247 ; 95 \%$ CI $22.2 \%-27.6 \%)$. Table 2 shows the distribution of subjects having $0-4$ SIRS criteria present at triage and their corresponding mean hours treated in the ED, ED cost, and hospital admission rates as well as the mean number of days of hospitalization and total inpatient hospital charges for those who were admitted. There was an overall difference in mean age between 
Table 1. Demographics of Participants and Nonparticipants

\begin{tabular}{|c|c|c|c|}
\hline Variable & Participants $(\mathrm{N}=991)$ & Nonparticipants $(\mathrm{N}=110)$ & P-Value \\
\hline Age, years (SD) & $47.0(20.1)$ & $40.2(15.6)$ & $\leq 0.001$ \\
\hline Male gender, N (\%) & $463(46.7)$ & $55(50.0)$ & NS \\
\hline White ethnicity, $\mathrm{N}(\%)^{*}$ & $135(13.8)$ & $18(16.4)$ & NS \\
\hline Hospital admissions, N (\%) & $203(10.5)$ & $6(5.6)$ & $\leq 0.001$ \\
\hline ED cost, dollars (SD) ${ }^{* *}$ & $441(358)$ & $279(212)$ & $\leq 0.001$ \\
\hline Inpatient costs, dollars (SD) ${ }^{* * * *}$ & $16,161(27,212)$ & $13,448(18,292)$ & NS \\
\hline \multicolumn{4}{|c|}{$\begin{array}{l}\text { ED, emergency department; SD, standard deviation. } \\
\text { "White versus nonwhite. } \\
\text { "**Analysis based on } 943 \text { and } 104 \text { participants and nonparticipants, respectively, for whom cost data were obtainable. } \\
{ }^{* * *} \text { Analysis based on } 202 \text { and } 6 \text { participants and nonparticipants, respectively, for whom service and procedure charges were obtainable. Inpatient cost is the sum of service and procedure charges. }\end{array}$} \\
\hline
\end{tabular}

Table 2. Linear Trends of ED LOS and Cost, Hospital Admissions, Hospital LOS, and Total Inpatient Costs by Number of SIRS Criteria and Pairwise Comparisons within ED LOS and Cost, Hospital LOS and Total Inpatient Cost by Number of SIRS Criteria

\begin{tabular}{|c|c|c|c|c|c|c|}
\hline $\begin{array}{c}\text { Number of SIRS } \\
\text { Criteria }\end{array}$ & $\mathbf{N}(\%)$ & ED LOS Hours (SD) & ED Cost Dollars (SD) & $\begin{array}{c}\text { Hospital Admissions } \\
\text { N (\%) }\end{array}$ & $\begin{array}{l}\text { Hospital LOS Days } \\
\text { (SD) }\end{array}$ & $\begin{array}{l}\text { Inpatient Cost Dollars } \\
\text { (SD) }\end{array}$ \\
\hline 0 Criteria & $395(39.9)$ & $5.8(4.7)$ & $351(289)$ & $44(11.1)$ & $3.8(2.7)$ & $10,996(11,992)$ \\
\hline 1 Criteria & $349(35.2)$ & $6.3(4.6)$ & $396(276)$ & $57(16.3)$ & $5.1(5.6)$ & $11,882(15,727)$ \\
\hline 2 Criteria & $175(17.6)$ & $8.0(6.1)$ & $448(34)$ & $59(33.7)$ & $7.5(12.6)$ & $16,214(19,518)$ \\
\hline 3 Criteria & $62(6.3)$ & $8.0(5.4)$ & $454(59)$ & $35(56.5)$ & $10.0(10.6)$ & $25,681(39,343)$ \\
\hline 4 Criteria & $10(1.0)$ & $8.6(6.0)$ & $606(192)$ & $8(80.0)$ & $11.4(14.1)$ & $43,973(78,221)$ \\
\hline P-value for Linear Trend & & $\leq 0.0001$ & $\leq 0.001$ & $\leq 0.001$ & $\leq 0.01$ & $\leq 0.01$ \\
\hline
\end{tabular}

ED: Emergency Department; LOS: Length of Stay; SD: Standard Deviation; SIRS: Systemic Inflammatory Response Syndrome.

the 5 SIRS groups (F Value of $6.38,4$ degrees of freedom, $\mathrm{p}<0.001$ ), and multiple comparison testing $(\mathrm{P}=0.5)$ showed that the mean age of patients with 2 SIRS criteria (52.1 years \pm 20.3 ) was significantly greater than those with no SIRS criteria (43.8 years \pm 18.8 ). There was a linear trend in the mean number of hours treated in the ED among the 5 SIRS criteria groups $(p<0.001)$. There was a $48.3 \%$ increase in the mean number of ED hours between subjects with no SIRS criteria and those with 4 SIRS criteria. There was a significant linear trend between mean ED cost and number of SIRS criteria present $(\mathrm{p}<0.001)$.

The proportion of hospitalized patients increased as the number of SIRS criteria increased $(\mathrm{p}<0.001)$ (Table 2$)$. The frequency of hospital admissions ranged between $11.1 \%$ for those with no SIRS criteria and $80.0 \%$ for those with 4 SIRS criteria. Among those with 2, 3, or 4 SIRS criteria, the proportion hospitalized were $33.7 \%, 56.5 \%$, and $80.0 \%$, respectively $(\mathrm{p}<0.001)$. Among hospitalized patients $(\mathrm{n}=203)$, a linear trend emerged between the mean number of hospitalization days and number of SIRS criteria, showing an overall increase of $200 \%$ in the mean length of stay (LOS) between those with no SIRS and those with 4 SIRS criteria $(\mathrm{p}<0.01)$. There was a significant linear trend between mean inpatient charges and the number of SIRS criteria $(\mathrm{p}<0.001)$. However, the linear relationship between service charges (hospital charges) and number of SIRS criteria $(\mathrm{p}<0.001)$ was stronger than the linear relationship between procedure charges (physician charges) and number of SIRS criteria $(\mathrm{p}=0.02)$.

Demographic, length of ED stay, and hospitalization differences between patients with and without SIRS are presented in Table 3. As Table 3 illustrates, SIRS patients were, on average, approximately 7 years older $(\mathrm{p}<0.001), 1.6$ times more likely to be white $(\mathrm{p}=0.03)$, treated 2 hours longer in the ED $(\mathrm{p}<0.001)$, and 4.5 times more likely to be admitted to the hospital $(\mathrm{p}<0.001)$. The total ED cost was $64 \%$ higher in those who presented with SIRS $(\mathrm{p}<0.001)$. Gender was not associated with SIRS disposition.

Table 4 focuses on the differences between SIRS and non-SIRS patients who were hospitalized $(n=203)$. There were differences in age, ethnicity, gender, and number of hours treated in the ED between patients with SIRS and without SIRS. ED charges were significantly different between the groups ( $\$ 956 \pm \$ 447$, SIRS; $\$ 705 \pm \$ 318$, nonSIRS; $\mathrm{p}<0.001)$. However, SIRS patients were hospitalized, on average, 4.2 times longer than non-SIRS patients $(\mathrm{p}=0.004)$. A statistically borderline difference was shown with respect to the type of admitting unit. SIRS patients were 2.2 times more likely to be admitted to an intensive care unit (ICU) than non-SIRS patients $(\mathrm{p}=0.06)$. There was a significantly greater number of deaths among SIRS patients compared with non-SIRS patients $(8.0 \%$ versus $1.0 \%, \mathrm{p}=0.04)$. Of note, SIRS patients consumed more hospital resources, as reflected by service charges for hospitalization $(\$ 30,305 \pm \$ 3000$, SIRS; $\$ 11,362 \pm \$ 1130$, non-SIRS; $\mathrm{p}<0.002)$, while incurring similar procedural charges $(\$ 5326$ \pm \$527, SIRS; $\$ 3204 \pm \$ 320$, non-SIRS; $\mathrm{p}=0.067$ )

Table 5 lists the sensitivity, specificity, and positive and negative predictive values for the prediction of hospital admission according to the number of SIRS criteria upon presentation to the ED. Of the 203 patients presenting with at least 2 SIRS criteria, 102 patients were hospitalized, yielding a sensitivity of $50.3 \%$. Sensitivity in predicting hospitalization decreased, whereas the specificity and positive predictive values increased as the number of SIRS criteria increased. Using a cutoff of 3 SIRS criteria and 4 SIRS criteria to predict hospitalization led to positive predictive values of $59.7 \%$ and $80 \%$, respectively. Specificity was maximized using a cutoff of 4 SIRS criteria.

ED discharge diagnoses were categorized into 3 disease groupings, including infectious, traumatic, or NINT groups. Table 6 lists the characteristics of these groups. The following significant overall differences emerged among the 3 groups: proportion of patients with SIRS $(p<0.05)$, hours spent in the ED $(p<0.001)$, the proportion of hospital admissions $(\mathrm{p}<0.01)$, and the number of inpatient days among those admitted $(p<0.0001)$. However, pairwise comparisons using analysis of variance showed that for the length of hours spent in the ED and LOS in the hospital, significant difference existed between the infectious and NINT groups only. There were no significant pairwise differences between the infectious and traumatic groups.

Table 7 describes the disposition of patients at ED presentation by SIRS status among the 3 disease categories. Among patients with an infectious etiology, SIRS patients were, on average, 5.1 years older 
Table 3. Characteristics of SIRS and Non-SIRS Patients

\begin{tabular}{|c|c|c|c|c|}
\hline Variable & SIRS Patients $(n=247)$ & Non-SIRS Patients $(n=744)$ & Odds Ratio (95\% CI) & P Value \\
\hline Age, years (SD) & $51.9(20.4)$ & $45.3(19.7)$ & - & $\leq 0.001$ \\
\hline \multicolumn{5}{|l|}{ Ethnicity, N (\%) } \\
\hline Nonwhite & $203(82.3)$ & $653(87.8)$ & & \\
\hline White & $44(17.7)$ & $91(12.2)$ & $1.6(1.1,2.3)$ & 0.03 \\
\hline \multicolumn{5}{|l|}{ Gender, N (\%) } \\
\hline Female & $134(54.3)$ & $394(53.0)$ & & \\
\hline Male & $113(45.7)$ & $350(47.0)$ & $0.9(0.7,1.3)$ & NS \\
\hline ED LOS, hours (SD) & $8.0(5.9)$ & $6.0(4.6)$ & - & $\leq 0.001$ \\
\hline ED cost, dollars (SD) & $463(30)$ & $283(11)$ & - & $\leq 0.001$ \\
\hline \multicolumn{5}{|c|}{ Hospital admission, N (\%) } \\
\hline No & $145(58.7)$ & $643(86.4)$ & & \\
\hline Yes & $102(41.3)$ & $101(13.6)$ & $4.5(3.2,6.2)$ & $\leq 0.001$ \\
\hline
\end{tabular}

Table 4. Characteristics of Hospitalized SIRS and Hospitalized Non-SIRS Patients (Numbers don't quite add up)

\begin{tabular}{|c|c|c|c|c|}
\hline Variable & SIRS Patients $(n=102)$ & Non-SIRS Patients $(n=101)$ & Odds Ratio (95\% CI) & P Value \\
\hline Age, years (SD) & $61.2(17.5)$ & $61.4(18.4)$ & - & NS \\
\hline \multicolumn{5}{|l|}{ Ethnicity, N (\%) } \\
\hline Nonwhite & $77(75.5)$ & $78(77.2)$ & & \\
\hline White & $25(24.5)$ & $23(22.8)$ & $1.1(0.6-2.9)$ & NS \\
\hline \multicolumn{5}{|l|}{ Gender, N (\%) } \\
\hline Female & $49(48.0)$ & $61(60.4)$ & & \\
\hline Male & $53(52.0)$ & $40(39.6)$ & $1.6(0.9-2.9)$ & NS \\
\hline ED LOS, hours (SD) & $9.3(6.4)$ & $8.8(4.7)$ & - & NS \\
\hline ED cost, dollars (SD) & $956(447)$ & $705(318)$ & - & $\leq 0.001$ \\
\hline Hospital LOS, days (SD) & $8.7(12.0)$ & $4.5(4.6)$ & -0.004 & \\
\hline \multicolumn{5}{|l|}{ Admitting unit, $\mathrm{N}(\%)$} \\
\hline Non-ICU & $84(82.4)$ & $92(91.1)$ & & \\
\hline ICU & $18(17.6)$ & $9(8.9)$ & $2.2(0.9-5.1)$ & 0.06 \\
\hline Service charge, dollars (SD) & $30,305(3000)$ & $11,362(1130)$ & - & 0.002 \\
\hline Procedure charge, dollars (SD) & $5326(527)$ & $3204(320)$ & - & 0.067 \\
\hline \multicolumn{5}{|l|}{ Hospital deaths" } \\
\hline No & $92(92.0)$ & $99(99.0)$ & & \\
\hline Yes & $8(8.0)$ & $1(1.0)$ & $8.6(1.1,386)$ & 0.04 \\
\hline
\end{tabular}

"Survival (yes|no) of 2 SIRS and 1 non-SIRS hospitalized patients were not obtained. For this analysis, Fisher's chi-square test was used and exact odds ratio and $95 \%$ CIs were calculated.

Table 5. Screening Performance of Hospital Admission Predictions

\begin{tabular}{|c|c|c|c|}
\hline Predictions of Hospital Admission & Number Admitted & $\%$ & 95\% Confidence Interval \\
\hline \multicolumn{4}{|l|}{$\geq 2$ SIRS criteria } \\
\hline Sensitivity & $102 / 203$ & 50.3 & $43.4-57.1$ \\
\hline Specificity & $643 / 788$ & 81.6 & $78.9-84.3$ \\
\hline Positive predictive value & $102 / 247$ & 41.3 & $35.2-47.4$ \\
\hline Negative predictive value & $643 / 744$ & 86.4 & $84.0-89.0$ \\
\hline \multicolumn{4}{|l|}{$\geq 3$ SIRS criteria } \\
\hline Sensitivity & $43 / 203$ & 21.2 & $15.6-26.8$ \\
\hline Specificity & $759 / 788$ & 96.3 & $95.0-97.6$ \\
\hline Positive predictive value & $43 / 72$ & 59.7 & $48.4-71.1$ \\
\hline Negative predictive value & $759 / 919$ & 82.6 & $80.1-85.0$ \\
\hline \multicolumn{4}{|l|}{$\geq 4$ SIRS criteria } \\
\hline Sensitivity & $8 / 203$ & 3.9 & $1.3-6.6$ \\
\hline Specificity & $786 / 788$ & 99.8 & $99.0-100$ \\
\hline Positive predictive value & $8 / 10$ & 80.0 & $55.2-100$ \\
\hline Negative predictive value & $786 / 981$ & 80.1 & $77.6-82.6$ \\
\hline
\end{tabular}


Table 6. Characteristics of and Differences Between Patients Presenting to the ED Related to Infection, Trauma, and Noninfectious-Nontraumatic (NINT) Etiology*

\begin{tabular}{|c|c|c|c|c|}
\hline & Infectious Etiology & Traumatic Etiology & NINT Etiology & P-value \\
\hline & $\mathrm{N}=\mathbf{2 8 1}$ & $N=144$ & $\mathrm{~N}=562$ & \\
\hline Age, years (SD) & $42.4(20.0)$ & $46.5(20.9)$ & $49.3(19.5)$ & NS \\
\hline Male gender (\%) & 44.8 & 47.9 & 47.3 & NS \\
\hline White race $(\%)$ & 11.4 & 16.7 & 13.9 & NS \\
\hline Patients with SIRS (\%) & 29.5 & 18.8 & 24.0 & $\leq 0.05$ \\
\hline Hours in $\mathrm{ED}^{+}(\mathrm{SD})$ & $5.7(4.0)$ & $5.9(4.7)$ & $7.1(5.5)$ & $\leq 0.001$ \\
\hline Hospital admission $(\%)^{++}$ & 19.6 & 10.4 & 23.5 & $\leq 0.01$ \\
\hline ICU admission ${ }^{++}$ & 16.6 & 13.3 & 12.1 & NS \\
\hline Days in hospital (SD) $)^{+++}$ & $6.7(5.5)$ & $10.3(22.0)$ & $6.0(8.1)$ & $\leq 0.0001$ \\
\hline
\end{tabular}

Table 7. Disposition of Patients: SIRS Status by ED Presentation Diagnosis (Infectious, Traumatic, and Noninfectious Nontraumatic [NINT])

\begin{tabular}{|c|c|c|c|c|c|c|c|c|c|}
\hline & \multicolumn{3}{|c|}{ Infectious Etiology } & \multicolumn{3}{|c|}{ Traumatic Etiology } & \multicolumn{3}{|c|}{ NINT Etiology } \\
\hline & Non-SIRS & SIRS & P-value $^{+}$ & Non-SIRS & SIRS & P-value $^{+}$ & Non-SIRS & SIRS & P-value ${ }^{+}$ \\
\hline & $\mathrm{N}=198$ & $\mathrm{~N}=83$ & & $\mathrm{~N}=117$ & $\mathrm{~N}=27$ & & $\mathrm{~N}=427$ & $\mathrm{~N}=135$ & \\
\hline Age, years (SD) & $40.9(19.7)$ & $46.0(20.6)$ & $\leq 0.05$ & $45.8(20.5)$ & $49.4+22.6$ & NS & $47.2(12.2)$ & $56(19.0)$ & $\leq 0.001$ \\
\hline Male (\%) & 45.0 & 44.6 & NS & 45.3 & 59.3 & NS & $48.2 \%$ & 45.4 & NS \\
\hline White race $(\%)$ & 11.6 & 10.8 & NS & 16.2 & 18.5 & NS & 11.5 & 21.5 & $\leq 0.01$ \\
\hline ED hours (SD) & $5.1(3.6)$ & $7.0(4.5)$ & $\leq 0.001$ & $5.5(4.2)$ & $5.5(4.2)$ & $\leq 0.05$ & $6.6(5.1)$ & $8.7(6.5)$ & $\leq 0.001$ \\
\hline Hospital admissions (\%) & 10.1 & 42.2 & $<0.001$ & 6.8 & 25.9 & $<0.05$ & 17.1 & 43.7 & $\leq 0.001$ \\
\hline ICU admissions (\%) & 10.0 & 20 & NS & 0 & 28.6 & - & 9.6 & 15.3 & NS \\
\hline Hospitalized days* (SD) & $4.5(3.0)$ & $7.8(6.3)$ & $\leq 0.05$ & $3.4(2.0)$ & $18.2(31.5)$ & NS & $4.7(5.2)$ & $7(10.6)$ & NS \\
\hline \multicolumn{10}{|c|}{$\begin{array}{l}\text { ED: Emergency Department; ICU: Intensive Care Unit; SD: Standard Deviation; SIRS: Systemic Inflammatory Response Syndrome } \\
{ }^{+} \text {Within group comparisons. } \\
\text {-Unable to statistically test differences. }\end{array}$} \\
\hline
\end{tabular}

$(\mathrm{p}<0.05)$. SIRS patients were also older by approximately 9 years among those with NINT etiology $(\mathrm{p}<0.001)$. There were no differences in gender between those who had SIRS and those who did not have SIRS within each disease group. Regarding race, only the NINT group displayed a preponderance of patients who were white with SIRS $(21.5 \%$ SIRS vs. $11.5 \%$ non-SIRS, $\mathrm{p}<0.01)$. SIRS patients spent significantly more hours receiving treatment in the ED than non-SIRS patients for each group; the infectious, traumatic, and NINT SIRS patients spent an average of 1.9, 2.3, and 2.1 more hours, respectively, than non-SIRS patients in the corresponding groups.

For each of the 3 groups, the prevalence of hospital admissions was significantly higher among SIRS patients compared to non-SIRS patients. Infectious SIRS patients were 6.5 times more likely (95\% CI, 3.4-12.2) to be admitted than their non-SIRS counterparts. Traumatic SIRS patients were 4.8 times more likely (95\% CI, 1.6-14.6) to be admitted than the non-SIRS traumatic patients. The NINT patients were 3.8 times more likely (95\% CI, 2.5-5.7) to be hospitalized than their non-SIRS counterparts. The number of hospitalized days significantly differed between the SIRS and non-SIRS patients within the infectious group only, with the SIRS patients spending, on average, 3.3 days longer.

\section{Discussion}

The 1992 ACCP/SCCM consensus conference developed broad definitions of sepsis in an attempt to aid the clinician in the early identification of patients at risk for sepsis who may benefit from novel therapies and to standardize the definitions for use in research protocols of these therapies [1].
These definitions have been incorporated in studies performed in the inpatient hospital setting, both in the intensive care unit and the general inpatient ward. However, this strategy selects for those patients who are already known to be acutely ill. The purpose of this study was to test the predictive characteristics of SIRS in the setting of ED triage, where little is known a priori about the patient's acuity.

This cross-sectional study was performed on all patients presenting to the ED of our institution over a 2-week period. Approximately onequarter of all patients met criteria for SIRS upon presentation. Still, the SIRS prevalence in this ED study is much lower than that seen in the inpatient population. In a study of 3708 patients conducted on general wards as well as the ICU, for example, the incidence of SIRS was $68 \% .3$ In a study of 170 ICU patients, 93\% met criteria for SIRS [10].

When limited to patients with suspected infection, the prevalence of SIRS appears to be much higher. These findings are intuitive, as one would suspect higher severity of illness in the inpatient setting and in those with an infectious etiology. However, to our knowledge, this is the first prospective study describing SIRS in an undifferentiated ED population.

The data from this study are in concordance with that of other studies in the inpatient setting, which have shown that patients who manifest SIRS have higher severity of illness as compared to controls $[3,10]$.

In this study, $43.5 \%$ (56.5\% admitted) of patients with 3 of 4 SIRS criteria and $20 \%$ ( $80 \%$ admitted) meeting 4 of 4 SIRS criteria were discharged home. One of the 2 patients discharged with 4 SIRS criteria returned within 8 hours and was admitted to the ICU while the remaining patient was lost to follow-up. 
Others have shown a temporal progression from SIRS to sepsis to severe sepsis to septic shock [3]. The above data suggests that SIRS comprises a continuum of illness. In the setting of infection, this continuum can occur prior to or along with a similar spectrum of illness beginning with sepsis and progressing through severe sepsis, septic shock, and multi-organ dysfunction syndrome. Why some SIRS patients recover and others progress in their disease process is currently unclear. More longitudinal study of this population may shed some light on this issue.

This study has shown that increasing numbers of SIRS criteria are associated with an increased length of ED stay and ED cost resulting from increased medical care requirements (Table 2). Thus, simply categorizing ED patients by SIRS criteria may provide a unique scoring system to determine ED staffing/support requirements. This may also provide a strategy for more rapid ED triage of critically ill patients. To date, there are no scoring systems to allow for the assessment and comparison of ED patients' severity of illness.

Likewise, this same SIRS scoring system predicts increased hospital LOS and inpatient cost, allowing for early identification of highcost admissions. Strategies and studies can be developed to test early therapeutic interventions in these higher cost patients to improve outcome and decrease inpatient costs and LOS. In comparing all SIRS patients to those without SIRS, these trends continued whether patients were hospitalized or not. Specifically, SIRS patients require longer ED LOS and incurred greater costs as well as longer inpatient LOS and higher costs. SIRS patients were twice as likely to be admitted to an ICU than non-SIRS patients, emphasizing the differences in severity of illness between these 2 groups. Lastly, this data shows that SIRS patients were more likely to die during hospitalization. SIRS thus indicates disease requiring longer and more expensive ED and, if admitted, hospital care.

SIRS has been mainly described in the setting of infection or trauma. Our data shows a significantly higher admission rate in SIRS patients with these diagnoses. Patients in the NINT group of our study similarly showed a higher admission rate if they met SIRS criteria. The NINT group includes patients with pancreatitis and burn injury, but the majority had a wide spectrum of diagnoses. It is clear that other disease states evoke the SIRS response and, when identified, require careful attention and possible hospitalization. Further study of this group is certainly warranted.

The finding that $59 \%$ of patients presenting to the ED meeting criteria for SIRS were discharged home requires further discussion. Previously healthy patients manifesting SIRS can, for the most part, be managed on an outpatient basis. For example, the patient with a diagnosis of acute pharyngitis, lobar pneumonia, or pyelonephritis may be discharged home in the majority of cases with few, if any, adverse outcomes. This is quite different from patients already admitted to the hospital with SIRS, a population which has been previously described.

Clinical experience demonstrates that numerous infected, even "septic" patients do not present manifesting SIRS. These include the elderly, those with significant comorbid factors (e.g., renal failure, immunosuppressive states, and congestive heart failure), and others who may fail to mount an appropriate inflammatory response to such insults. Medications such as beta-blockers, corticosteroids, and overthe-counter anti-inflammatory medications may further mask the presentation of SIRS. Lastly, patients may present at various intervals after the onset of symptoms, and therefore, may present manifesting a predominantly "anti-inflammatory" response (i.e., compensatory anti-inflammatory response syndrome or mixed antagonistic response syndrome) that lacks SIRS criteria [6].

A major limitation of this study is that SIRS was determined at a single point in time, upon entry to the health care system, and not followed within the ED after treatment or during hospitalization. While one of the shortcomings of this study was that these patients were not followed after discharge, the authors' clinical experience suggests that few, if any, adverse outcomes ensue. Quality assurance programs did not signal an inordinate number of ED readmissions during, or immediately after, the study period. This, along with data showing poor sensitivity and positive predictive value, leads to the conclusion that while SIRS may be a good indicator of severe illness for the inpatient population, it is a much weaker indicator in the outpatient or emergency setting. In spite of the discussed limitations, this data shows that most individuals without SIRS were not admitted to the hospital. The presence of SIRS has low sensitivity for admission and thus is only a marker of potentially serious underlying disease.

In order to better assess disease severity so as to optimize interventions, further studies will need to be done to identify patients with or without SIRS by disease state, length of illness, comorbidities, age, race, and medications taken. With this approach, SIRS may be useful as an indicator of individual disease severity, but this approach results in yet another scoring system. It is becoming more realistic that in the future these assessments will include serum biomarkers (e.g., cytokines). This combination of individualized clinical assessments and objective biomarker profiles will hopefully allow tailor-made therapies, possibly including immune modulation therapy.

In this study population, SIRS has poor sensitivity and fair specificity for requiring hospitalization. While this may not be the outcome measure envisioned by the ACCP/SCCM consensus conference, it is doubtful that the conference members would wish to employ novel therapies for sepsis on outpatients. However, when combined with other data, SIRS, or its absence, may help in the accurate estimation of disease severity and in predicting clinical outcome.

\section{Conclusions}

SIRS is commonly present in patients presenting to the ED. Its presence indicates a higher frequency of serious disease as reflected by ED LOS and cost and inpatient LOS and cost. Its presence may reliably indicate a population's potential for serious disease states. However, by itself, SIRS is not sensitive or specific enough in the ED setting to identify serious disease progression and most likely will need to be combined with biomarkers in future studies.

\section{References}

1. American College of Chest Physicians/Society of Critical Care Medicine Consensus Conference (1992) Definitions for sepsis and organ failure and guidelines for the use of innovative therapies in sepsis. Crit Care Med 20: 864-874.

2. Bone RC (1992) Toward an epidemiology and natural history of SIRS (systemic inflammatory response syndrome). JAMA 268: 3452-3455.

3. Rangel-Frausto MS, Pittet D, Costigan M, Hwang T, Davis CS, et al. (1994) The natural history of the systemic inflammatory response syndrome (SIRS). A prospective study. JAMA 273: 117-123

4. Bone RC (1994) Sepsis and SIRS. Nephrol Dial Transplant 4: 99-103.

5. Salvo I, de Cian W, Musicco M, Langer M, Piadena R, et al. (1995) The Italian SEPSIS study: preliminary results on the incidence and evolution of SIRS, sepsis, severe sepsis and septic shock. Intensive Care Med 2: 244-249.

6. Bone RC (1996) Sir Isaac Newton, sepsis, SIRS, and CARS. Crit Care Med 24: 11251128 . 
Bissonette AH (2018) Systemic Inflammatory Response Syndrome (SIRS) in the emergency department: An original investigation - A look back in time

7. Smail N, Messiah A, Edouard A, Descorps-Declère A, Duranteau J, et al. (1995) Role of systemic inflammatory response syndrome and infection in the occurrence of early multiple organ dysfunction syndrome following severe trauma. Intensive Care Med 21: $813-816$.

8. Bone RC (1996) Toward a theory regarding the pathogenesis of the systemic inflammatory response syndrome: what we do and do not know about cytokine regulation. Crit Care Med 24: 163-172.
9. Cremer J, Martin M, Redl H, Bahrami S, Abraham C, et al. (1996) Systemic inflammatory response syndrome after cardiac operations. Ann Thorac Surg 61: 17141720. [Crossref]

10. Pittet D, Rangel-Frausto S, Li N, Tarara D, Costigan M, et al. (1995) Systemic inflammatory response syndrome, sepsis, severe sepsis and septic shock: incidence, morbidities and outcomes in surgical ICU patients. Intensive Care Med 21: 302309.

Copyright: $\bigcirc 2018$ Bissonette AH. This is an open-access article distributed under the terms of the Creative Commons Attribution License, which permits unrestricted use, distribution, and reproduction in any medium, provided the original author and source are credited. 